\title{
ON THE EXISTENCE OF ACYCLIC CURVES SATISFYING CERTAIN CONDITIONS WITH RESPECT TO A GIVEN CONTINUOUS CURVE*
}

\author{
BY \\ C. M. CLEVELAND
}

Part I of this paper has to do with connected sets of cut points $\dagger$ of a given continuous curve. It is shown that, in the plane, any two points belonging to a connected set $K$ of cut points of a given continuous curve $M$ lie together in an arc which is common to $K$ and a point set consisting of the boundaries of a finite number of complementary domains of $M$. G. T. Whyburn $\ddagger$ calls attention to the fact that from his results it follows that $K$ is arcwise connected. To show that any two points of $K$ can be joined by an arc which is common to $K$ and a point set consisting of the boundaries of a finite number of complementary domains of $M$, is the object of Part I. Part II has to do with a totally disconnected closed subset $K$ of a given plane continuous curve $M$ no subset of which disconnects $M$. R. L. Moore $\S$ has shown that, in the plane, any two points not belonging to a bounded continuous curve can be joined by an arc which does not disconnect the continuous curve. The object of Part II of this paper is to show that if, in the plane, $M$ is a bounded continuous curve which contains no domain, and $K$ is a closed and totally disconnected subset of $M$, such that no subset of $K$ disconnects $M$, then there exists an acyclic continuous curve $T$, containing $K$, such that (1) all the end points $\|$ of $T$ belong to $K$ and (2) the point set $M \cdot T$ is totally disconnected and $M-T$ is connected.

I wish to acknowledge my indebtedness to Professor R. L. Moore, not only for the suggestions of the problems treated, but also for his many helpful criticicms in their solutions. To him is due, in a large measure, the success

* Presented to the Society, in part, June 12, 1926, and September 9, 1930; received by the editors December 22, 1930.

$\dagger$ A point $P$ is said to be a cut point of a continuum $M$ if $M-P$ can be expressed as the sum of two mutually separated sets. p. 176.

$\ddagger$ Concerning the structure of a continuous curve, American Journal of Mathematics, vol. 50 (1928),

$\S$ Concerning paths that do not separate a given continuous curve, Proceedings of the National Academy of Sciences, vol. 12 (1926), pp. 745-753.

$\|$ The term end point will be used in the sense as defined by R. L. Wilder, Concerning continuous curves, Fundamenta Mathematicae, vol. 7 (1925), p. 358, i.e., a point $P$ of a continuous curve $M$ is an end point of $M$ provided it is true that if $t$ is an arc of $M$ having $P$ as one of its extremities, then $M-(t-P)$ contains no connected subset which contains $P$. 
of these investigations. His stimulating personality has been a source of constant encouragement to me in the study of mathematics.

\section{PART I}

LEMMA I. If $K$ is a connected set of points belonging to a continuous curve $M$, and $J$ is the outer boundary of a complementary domain $D$ of $M$, and $M_{1}$ is a component of $M-J$ which lies within $J$, then $K \cdot \bar{M}_{1}$ is connected.

By a theorem of R. L. Moore, $\dagger J$ contains exactly one limit point $P$ of $M_{1}$ and thus $\bar{M}_{1}=M_{1}+P$. The closed point sets $M_{1}+P$ and $M-M_{1}$ have only the point $P$ in common and their sum is $M$. Hence, unless $K=K \cdot \bar{M}_{1}$, then $K-P$ is the sum of the two mutually separated point sets $K \cdot M_{1}$ and $K\left(M-M_{1}\right)$. Therefore $K \cdot M_{1}+P$ is connected. But $K \cdot M_{1}+P=K \cdot \bar{M}_{1}$. Hence $K \cdot \bar{M}_{1}$ is connected.

Lемма II. If $X$ is a point belonging to the boundary of a complementary domain $D$ of a continuous curve $M$, then there do not exist infinitely many simple closed curves of $M$, each of which encloses $X$ and is the outer boundary of a complementary domain of $M$.

Lemma II follows from the fact that the complementary domains of $M$ form a contracting sequence, $\ddagger$ and the fact that no two complementary domains of $M$ have the same outer boundary. $\S$

Lемма III. Suppose (1) $K$ is a connected set of points belonging to a continuous curve $M$, (2) $D_{1}, D_{2}, D_{3}, \cdots$ are all the bounded complementary domains of $M$ such that for each $i, \bar{D}_{i}$ contains a point of $K$, (3) for each $i, J_{i}$ is the outer boundary of $D_{i}$, and (4) $J_{1}{ }^{*}, J_{2}{ }^{*}, J_{3}{ }^{*}, \ldots$ is a finite or infinite subsequence of the sequence $J_{1}, J_{2}, J_{3}, \cdots$. Then the set $K^{*}$ consisting of all points $X$ of $K$ such that $X$ is not interior to $J_{i}{ }^{*}$ for any value of $i$ is connected, and if all but a countable number of the points of $K$ are cut points of $M$, and no point of $K^{*}$ is interior to $J_{i}$ for any value of $i$, and $K^{*}$ contains more than one point, then $K^{*}$ is a subset of the boundary of the unbounded complementary domain of $M$.

Suppose there exists a subsequence $J_{1}^{*}, J_{2}^{*}, J_{3}^{*}, \ldots$ of the sequence $J_{1}, J_{2}, J_{3}, \cdots$ for which the set $K^{*}$ consisting of all points $X$ of $K$, such that

$\dagger$ Concerning paths that do not separate a given continuous curve, loc. cit., Theorem 6.

$\ddagger$ If $H$ is a sequence of point sets and for each positive number $e$ only a finite number of point sets of the set $H$ are of diameter greater than $e$, then $H$ is said to be $a$ contracting sequence of point sets. See R. L. Moore, Concerning upper semi-continuous collections, Monatshefte für Mathematik und Physik, vol. 36 (1929), pp. 81-88. See also R. L. Moore, Report on continuous curves from the viewpoint of analysis situs, Bulletin of the American Mathematical Society, vol. 29 (1923).

$\S$ See R. L. Moore, Concerning paths that do not separate a given continuous curve, loc. cit., Theorem 1 . 
$X$ is not interior to $J_{i}^{*}$ for any value of $i$, is not connected. The set $K^{*}$ can be expressed as the sum of two mutually separated sets $K_{1}$ and $K_{2}$. It follows from Lemma II that there exists a subsequence $J_{1}^{* *}, J_{2}^{* *}, J_{3}^{* *}, \ldots$ of the sequence $J_{1}^{*}, J_{2}^{*}, J_{3}^{*}, \cdots$ such that (1) if $X$ is any point lying on or within some curve of the sequence $J_{1}^{*}, J_{2}^{*}, J_{3}^{*}, \cdots$, then $X$ lies on or within some curve of the sequence $J_{1}^{* *}, J_{2}^{* *}, J_{3}^{* *}, \cdots$, (2) for $m \neq n, J_{m}^{* *}$ contains no point interior to $J_{n}^{* *}$. For each positive integer $n$, and for each point $X$ of $K_{1}$ belonging to $J_{n}^{* *}$, add to $K_{1}$ every point $Y$ of $K$ such that $Y$ belongs to some component of $M-J_{n}^{* *}$ which lies within $J_{n}^{* *}$ and has $X$ as a limit point. Let $K_{1}^{*}$ denote the resulting set. For each positive integer $n$ and for each point $X$ of $K_{2}$ belonging to $J_{n}^{* *}$ add to $K_{2}$ every point $Y$ of $K$ such that $Y$ belongs to some component of $M-J_{n}^{* *}$ which lies within $J_{n}^{* *}$ and has $X$ as a limit point. Let $K_{2}^{*}$ denote the resulting set. Since $K$ is the sum of the sets $K_{1}^{*}$ and $K_{2}^{*}$, one of these sets contains a limit point of the other. We shall consider the case where $K_{1}^{*}$ contains a limit point of $K_{2}^{*}$. Let $P$ denote one such limit point. The point $P$ belongs to $K_{1}$, for suppose it does not. There exists a positive integer $t$ such that $J_{t}^{* *}$ encloses $P$. Only a finite number of components of $M-J_{t}^{* *}$ are of diameter greater than a positive number, $\dagger$ and all the points of $K$ belonging to that component of $M-J_{t}^{* *}$ which contains $P$ belong also to $K_{1}^{*}$. It follows that $P$ is not a limit point of $K_{2}^{*}$ contrary to hypothesis. Hence $P$ belongs to $K_{1}$. It follows then that $P$ is the sequential limit point of a sequence $P_{1}, P_{2}, P_{3}, \ldots$ of points belonging to the set $K_{2}^{*}-K_{2}$ such that for $i \neq j, P_{i}$ and $P_{j}$ belong to different components of $M-\left(J_{1}^{* *}+J_{2}^{* *}+J_{3}^{* *}+\cdots\right)$. For each positive integer $n$ let $Z_{n}$ denote the limit point of that component of $M-\left(J_{1}^{* *}+J_{2}^{* *}+J_{3}^{* *}+\cdots\right)$ which contains $P_{n}$. Since (1) for each $n$ the point $Z_{n}$ belongs to $K_{2}$, (2) the curves $J_{1}^{* *}$, $J_{2}^{* *}, J_{3}^{* *}, \cdots$ form a contracting sequence, $\ddagger$ and (3) not more than a finite number of components of $M-J_{i}^{* *}$, for any value of $i$, are of diameter greater than a positive number, it follows that $P$ is a limit point of $K_{2}$ and belongs to $K_{1}$, contrary to the assumption that $K_{1}$ and $K_{2}$ are mutually separated. Hence $K^{*}$ is connected and the supposition that a subsequence $J_{1}^{*}, J_{2}^{*}, J_{3}^{*}, \cdots$ of the sequence $J_{1}, J_{2}, J_{3}, \cdots$ exists for which the set of all points $X$ of $K$ such that $X$ is not interior to $J_{i}^{*}$ for any value of $i$ is not connected, has led to a contradiction.

Let $K^{* *}$ denote the set of all points $X$ of $K$ such that $X$ is not interior to $J_{i}$ for any value of $i$. Suppose $K^{* *}$ contains more than one point. If $Q$ denotes

$\dagger$ See W. L. Ayres, Concerning continuous curves and correspondences, Annals of Mathematics, (2), vol. 28 (1927), Theorem 1.

$\ddagger$ See R. L. Moore, Report on continuous curves from the viewpoint of analysis situs, loc. cit. 
a point of $K^{* *}$ which does not belong to the boundary $B^{*}$ of the unbounded complementary domain $D^{*}$ of $M$, let $C_{q}$ denote a circle with center at $Q$, such that $C_{q}$ encloses no point of $D^{*}$. By a theorem of G. T. Whyburn, $\dagger$ the set of all cut points $X$ of $M$ such that $X$ lies on some simple closed curve of $M$ is a countable set. But every cut point of $M$ is on the boundary of some complementary domain $\ddagger$ of $M$, and therefore either belongs to $B^{*}$ or lies on or within $J_{i}$ for some value of $i$. Hence, since $K^{* *}$ is connected and therefore contains uncountably many points within $C_{q}$, there are points of $K^{* *}$ interior to $C_{q}$ which belong also to $K-K^{* *}$, which is impossible. Therefore $K^{* *}$ is a subset of $B^{*}$.

THEOREM I. If $B$ is the boundary and $J$ the outer boundary of a bounded complementary domain $D$ of a continuous curve $M$, and $K$ is a connected set of cut points of $M$ such that $K$ contains a point in common with $J$, any point of $K$ which belongs to $B-J$ can be joined to some point of $J$ by an arc common to $K$ and to $B$.

Let $P$ denote a point of $K$ belonging to $B-J$, and let $M_{1}$ denote that component of $M-J$ which contains $P$. Let $Q$ denote the limit point $\S$ of $M_{1}$ belonging to $J$ and let $K^{*}$ denote the set $K \cdot \bar{M}_{1}$. Since $K$ is connected, $Q$ belongs to $K$. By Lemma I, $K^{*}$ is connected. Since $M_{1}+Q$ is a continuous curve, and $B\left(M_{1}+Q\right)$ is the boundary of the unbounded complementary domain of $M_{1}+Q$, by Lemma III the set $B \cdot K^{*}$ is connected. Hence, by a theorem of R. L. Wilder, $\| P$ can be joined to $Q$ by an arc belonging to $B$ and to $K^{*}$.

THEOREM II. If $J$ is the outer boundary of a bounded complementary domain $D$ of a continuous curve $M$, and $K$ is a connected set of cut points of $M$ containing a point in common with $J$, then any point of $K$ within $J$ can be joined to some point of $J$ by an arc which is common to $K$ and to a point set consisting of the boundaries of a finite number of complementary domains of $M$.

Let $P$ denote a point of $K$ within $J$. By Lemma II, if $G$ denotes the collection of all simple closed curves of $M$ each of which encloses $P$ and is the outer boundary of some complementary domain of $M$, then $G$ is finite. Let $J_{1}, J_{2}$, $J_{3}, \cdots, J_{n}$ denote the curves of the collection $G$, where for each positive integer $i(i<n), J_{i}$ is a subset of the point set consisting of $J_{i+1}$ together with

$\dagger$ Concerning continua in the plane, these Transactions, vol. 29 (1927), pp. 369-400, Theorem 29.

$\ddagger$ See R. L. Moore, Concerning the common boundary of two domains, Fundamenta Mathematicae, vol. 6 (1924), pp. 203-213. orem 6 .

$\S$ See R. L. Moore, Concerning paths that do not separate a given continuous curve, loc. cit., The-

|| Concerning continuous curves, Fundamenta Mathematicae, vol. 7, pp. 340-377, Theorem 20. 
its interior. Suppose $J_{m}$ is $J$. For each positive integer $k(k<m)$, let $D_{k}$ denote that complementary domain of $M$ whose outer boundary is $J_{k}$, let $M_{k}$ denote that component of $M-J_{k}$ which contains $P$, and let $Q_{k}$ denote the point of $J_{k}$ which is a limit point of $M_{k}$. Suppose there exists a positive integer $q(q<m)$ such that $Q_{q+1}$ does not belong to the boundary of $D_{q}$. Let $K_{q+1}$ denote the set of all points $X$ of $K$ such that (a) $X$ belongs to $K \cdot \bar{M}_{q+1}$, (b) the point $X$ is not interior to the outer boundary of any complementary domain of $M$ (except $D_{q+1}$ ) whose boundary contains a point of $K \cdot \bar{M}_{q+1}$. Since $J_{q+1}$ is the outer boundary of $D_{q+1}$, every cut point of $M$ which belongs to $M_{q+1}$ is also a cut point of $M_{q+1}$. By Lemmas I and III together with the fact that $\bar{M}_{q+1}$ is a continuous curve, $K_{q+1}$ is connected. But it contains $Q_{q}$ and $Q_{q+1}$ and is a subset of the boundary of $D_{q+1}$. By Wilder's theorem $\dagger$ there exists for each positive integer $i(i<m)$ an arc from $Q_{i}$ to $Q_{i+1}$ which is common to $K$ and the boundary of $D_{i+1}$. Hence there exists an $\operatorname{arc}$ from $P$ to a point of $J$ which is common to $K$ and a point set consisting of the boundaries of a finite number of complementary domains of $M$.

THEOREM III. If $K$ is a connected set of cut points of a continuous curve $M$, and $X$ and $Y$ are two points of $K$, then there exists an arc from $X$ to $Y$ which is common to $K$ and a point set consisting of the sum of the boundaries of a finite number of complementary domains of $M$.

Suppose $X$ belongs to the boundary $B^{*}$ of the unbounded complementary domain $\ddagger D^{*}$ of $M$. If $Y$ belongs to $B^{*}$ the proof of the theorem follows from Lemma III together with Wilder's theorem. $\$$ Suppose $Y$ does not belong to $B^{*}$. By Lemma II if $G$ denotes the collection of all simple closed curves of $M$ each of which encloses $Y$ and is the outer boundary of some complementary domain of $M$, then $G$ is finite. There exists a simple closed curve $J^{*}$ of the collection $G$ such that the point set consisting of the sum of all the simple closed curves of $G$ contains no point exterior to $J^{*}$. By Theorem II the point $Y$ can be joined to a point $Y_{1}$ of $J^{*}$ by an arc $Y Y_{1}$, and by Lemma III together with Wilder's theorem $\| Y_{1}$ can be joined to $X$ by an $\operatorname{arc} Y_{1} X$ such that each of the $\operatorname{arcs} Y Y_{1}$ and $Y_{1} X$ is common to $K$ and a point set consisting of the sum of the boundaries of a finite number of complementary domains of $M$. From the sum of the arcs $Y Y_{1}$ and $Y_{1} X$ there exists an arc from $X$ to $Y$ which satisfies the conditions of Theorem III.

$\dagger$ Concerning continuous curves, loc. cit.

$\ddagger$ Every other case may be reduced to this one by an inversion.

$\S$ Concerning continuous curves, loc. cit.

|| Ibid. 


\section{PART II}

Definition. An $\operatorname{arc} X Y$ will be said to have property $\alpha$ with respect to a continuous curve $M$ provided it satisfies the following conditions: (1) the arc $X Y$ contains no cut point of $M,(2)$ the common part of arc $X Y$ and $M$ is totally disconnected, (3) if the boundary $B$ of a complementary domain $D$ of $M$ contains two points $U$ and $V$ in common with arc $X Y$, then segment $U V$ is a subset of $D,(4)$ segment $X Y$ of the arc $X Y$ contains no point common to the boundaries $B_{1}$ and $B_{2}$ of two distinct complementary domains $D_{1}$ and $D_{2}$ of $M$ which is also a boundary point of some complementary domain of the point set $D_{1}+D_{2}+B_{1}+B_{2}$.

It is clear that if $X Y$ is an arc having property $\alpha$ with respect to a continuous curve $M$, and $Y Z$ is an arc which contains only the point $Y$ in common with $M$ or with arc $X Y$, and if $X Z$, the sum of the $\operatorname{arcs} X Y$ and $Y Z$, satisfies properties (3) and (4) of the preceding definition, then arc $X Z$ has property $\alpha$ with respect to $M$.

Theorem IV. If $M$ is a bounded continuous curve, and $K$ is a closed and totally disconnected subset of $M$ whose omission leaves $M$ connected and such that $K$ belongs to the boundary $B$ of a complementary domain $D$ of $M$, then there exists an acyclic continuous curve whose end points are identical with the set $K$ and which is a subset of $D+K$.

Since $B$ is a continuous curve, $\dagger$ by a theorem of R. L. Moore $\ddagger$ there exists a continuum $N$ containing $K$ and which is a subset of $D+K$. Let $N_{1}$ denote the continuum formed by adding to $N$ all the bounded complementary domains of $N$. Since $K$ is closed and totally disconnected, it follows that there exists a circle $C$ which is a subset of $D-N_{1}$. Let $T$ denote an inversion about $C$, let $M^{*}$ and $N_{1}{ }^{*}$ denote the images of $M$ and $N_{1}$ respectively under $T$, and let $M_{1}^{*}$ denote the continuum formed by adding to $M^{*}$ all the bounded complementary domains of $M^{*}$. It is clear that (a) $M_{1}^{*}-K$ is connected, (b) the set of points common to $M_{1}^{*}$ and $N_{1}^{*}$ is $K$, (c) neither $M_{1}^{*}$ nor $N_{1}^{*}$ separates the plane. Therefore by a theorem of R. L. Moore $\S$ there exists a simple closed curve $J$ which encloses $M_{1}^{*}-K$ but encloses no point of $N_{1}^{*}-K$ and contains $K$ but no point $\left(N_{1}^{*}+M_{1}^{*}\right)-K$. Let $P$ denote the center of $C$. If $J$ does not contain $P$ let $J^{*}$ denote $J$, and if $J$ contains $P$ let $J^{*}$ denote a

† See Marie Torharst, Über den Rand der einfach zusammenhängenden ebenen Gebiete, Mathematische Zeitschrift, vol. 9 (1921), pp. 45-65.

$\ddagger$ Some separation theorems, Proceedings of the National Academy of Sciences, vol. 13 (1927), pp. 711-716, Theorem I.

$\S$ Concerning the separation of point sets by curves, Proceedings of the National Academy of Sciences, vol. 11 (1925), pp. 469-476, Theorem 2. 
simple closed curve which contains $K$ but not $P$ and is a subset of $J$ plus a circle which encloses $P$ but which neither contains nor encloses any point of $N_{1}^{*}+M_{1}^{*}$. H. M. Gehman $\dagger$ has shown that if $E F$ is an arc containing a totally disconnected closed point set $L$, then $L$ is identical with the end points of an acyclic continuous curve which contains only the set $L$ in common with the arc $E F$. From this it follows that there exists an acyclic continuous curve $W$ whose end points are identical with $K$ and which is a subset of $K$ plus the exterior of $J^{*}$. The image $W^{*}$ of $W$ under the inverse of $T$ satisfies the conditions of Theorem IV.

THEOREM V. If $M$ is a bounded continuous curve, and $K$ is a closed point set, and $K^{*}$ is the set of all points $X$ such that $X$ belongs either to $K$ or to a point set containing a point of $K$ and consisting of a complementary domain of $M$ together with its boundary, then $K^{*}$ is closed.

Suppose $P$ is a limit point of $K^{*}$ which does not belong to $K^{*}$. The point $P$ belongs to $M$ and since $P$ does not belong to $K^{*}$ it is not a boundary point of any complementary domain $D$ of $M$ such that $D$ is a subset of $K^{*}$. Since $P$ does not belong to $K$, there exists a circle $C$ with center at $P$ such that $C$ neither contains nor encloses any point of $K$. Let $C^{*}$ denote a circle with center at $P$ and of diameter one-half that of $C$. It follows that there are infinitely many complementary domains of $M$ each of which contains a point exterior to $C$ and a point interior to $C^{*}$. This is impossible since the complementary domains of $M$ form a contracting sequence. $\ddagger$ Hence the suppositon that $P$ is a limit point of $K^{*}$ which does not belong to $K^{*}$ has led to a contradiction. Therefore $K^{*}$ is closed.

Theorem VI. Suppose $K$ is a closed and totally disconnected point set, and $M$ is a closed and bounded point set containing $K$ such that (a) the sum of all the components of $M$ which are not single points can be expressed as the sum of a countable number of continuous curves $C_{1}, C_{2}, C_{3}, \cdots$, not more than a finite number of which are of diameter greater than a positive number, (b) the set of all points $X$ such that $X$ belongs to at least two curves of the sequence $C_{1}, C_{2}, C_{3}, \cdots$ is a subset of $K$. Then each component of $M$ is a continuous curve and not more than a finite number of components of $M$ are of diameter greater than a positive number.

From the fact that a continuum which is not a continuous curve fails to be connected im kleinen at a continuum of points, $\ddagger$ and the fact that $K$ is rem 6.

† Concerning acyclic continuous curves, these Transactions, vol. 29 (1927), pp. 553-568, Theo-

$\ddagger$ See R. L. Moore, Report on continuous curves from the viewpoint of analysis situs, loc. cit. 
closed and totally disconnected, it follows that each component of $M$ is a continuous curve. Suppose there exists a positive number $e$ and an infinite sequence $M_{1}, M_{2}, M_{3}, \cdots$ of components of $M$ each of which is of diameter greater than $e$. There exists a subcontinuum $M^{*}$ of $M$ and a subsequence $M_{1}^{*}, M_{2}^{*}, M_{3}^{*}, \cdots$ of the sequence $M_{1}, M_{2}, M_{3}, \cdots$ having $M^{*}$ as sequential limiting set. $\dagger$ There exists a point $P$ belonging to $M^{*}-K$, and a positive integer $n$, such that, if $C$ and $C^{*}$ are circles with centers at $P$ and of diameters $e / n$ and $e /(2 n)$ respectively, then neither $C$ nor $C^{*}$ contains or encloses any point of $K$. Since there are infinitely many components of $M$ each containing a point exterior to $C$ and a point interior to $C^{*}$, there are infinitely many curves of the sequence $C_{1}, C_{2}, C_{3}, \ldots$ each of which is of diameter greater than $e /(4 n)$ contrary to hypothesis. Hence the supposition that there exists a positive number $e$ and an infinite sequence $M_{1}, M_{2}, M_{3}, \cdots$ of components of $M$ each of which is of diameter greater than $e$ has led to a contradiction.

THEOREM VII. If $M$ is a bounded continuous curve which contains no domain, and $T$ is an acyclic continuous curve having the properties (a) $T$ contains no cut point of $M,(\mathrm{~b})$ the set of all points common to $T$ and $M$ is totally disconnected, (c) if $D$ is any complementary domain of $M$, any two points of the set $T \cdot \bar{D}$ can be joined by an arc of $T$ which except for end points is a subset of $D$, then $M-T$ is connected.

Let $D$ denote a complementary domain of the continuous curve $M+T$ whose boundary $B$ contains a point of $T$. Let $D_{M}$ denote the complementary domain of $M$ which contains $D$, and let $B_{M}$ denote its boundary. Let $M_{1}$ denote the set $B-T$. Suppose $M_{1}$ is not connected. If $T$ contains only one point $A$ in common with $B_{M}$ the point $A$ is a cut point of $B_{M}$, and by a theorem of R. L. Moore $\ddagger A$ is a cut point of $M$ contrary to hypothesis. Hence there exists by property (c) a complementary domain $D_{\mathrm{i}}$ of $M+T$ distinct from $D$ such that $D_{1}$ is a subset of $D_{M}$. Let $J$ denote the outer boundary of $D$ with respect to $D_{1}$. By a theorem of R. L. Moore $\delta J$ is a simple closed curve. The curve $J$ contains a point of $T-M$ and a point of $M-T$. Suppose there exists a component $L$ of $M_{1}$ which contains no point of $J$. By a theorem of R. L. Moore\| the component $L^{*}$ of $B-J$ which contains $L$ has exactly one limit point $Q$ in $J$. Since $T$ contains no cut point of $M$ the point $Q$ does not belong to $T \cdot M$. If $Q$ belongs to $T$ let $Q_{1}$ denote a point of $L$ and let $Q_{2}$ de-

† See R. G. Lubben, Concerning limiting sets in abstract spaces, these Transactions, vol. 30, pp. 668-685.

$\ddagger$ Concerning the common boundary of two domains, loc. cit.

$\$$ Concerning continuous curves in the plane, Mathematische Zeitschrift, vol. 15 (1922).

\| Concerning paths that do not separate a given continuous curve, loc. cit., Theorem 6. 
note a point of $M_{1} J$. There exists an arc $\dagger$ of $M$ from $Q_{1}$ to $Q_{2}$. If $Q$ belongs to $M_{1}$, let $Q_{1}$ denote a point of $T$ belonging to $L^{*}$ and let $Q_{2}$ denote a point of $T$ belonging to $J$. There exists an arc of $T$ from $Q_{1}$ to $Q_{2}$. In either case the supposition that there exists a component of $M_{1}$ containing no point of $J$ contradicts the fact that $J$ is the outer boundary of $D$ with respect to $D_{1}$. Hence there exist arcs $a_{1}$ with end points $A_{1}$ and $A_{2}$ and $b_{1}$ with end points $B_{1}$ and $B_{2}$ such that arcs $a_{1}$ and $b_{1}$ belong to different components of $J \cdot M_{1}$. Let $s_{1}$ and $s_{2}$ denote the two arcs of $J$ from $A_{1}$ to $B_{1}$. Both $s_{1}$ and $s_{2}$ contain points of $T$. There exists an arc $\ddagger t$ of $T$ from a point in $s_{1}$ to a point in $s_{2}$. Let $t_{1}$ denote a subarc of $t$ having one end point $C_{1}$ in $s_{1}$ and one end point $C_{2}$ in $s_{2}$ and containing only the points $C_{1}$ and $C_{2}$ in common with $J$. Let $t_{2}$ denote an arc with $C_{1}$ and $C_{2}$ as end points and which except for $C_{1}$ and $C_{2}$ is a subset of $D$. Let $J_{1}$ denote the simple closed curve formed from the sum of the $\operatorname{arcs} t_{1}$ and $t_{2}$. The curves $J$ and $J_{1}$ have only the points $C_{1}$ and $C_{2}$ in common. Since the segment $t_{2}$ is a subset of $D$ and the segment $t_{1}$ contains no point in common with $D$, the point set $J-J_{1}$ is not a subset of a single complementary domain of $J_{1}$. Hence $a_{1}$ and $a_{2}$ belong to different complementary domains of $J_{1}$. Of the two points $C_{1}$ and $C_{2}$, if one belongs to $T-M$ the other is a cut point of $M$ contrary to hypothesis. If $C_{1}$ and $C_{2}$ both belong to $M$, by property (c) they do not both belong to the boundary of any complementary domain of $M$ except $D_{M}$. Hence $J-\left(C_{1}+C_{2}\right)$ is a subset of $D_{M}$. There exists an $\operatorname{arc} t_{3}$ in $D_{M}$ having one end point in $t_{1}$ and one end point in $t_{2}$ and which, except for end points, contains no point in common with $J_{1}$. The point set $J_{1}+t_{3}$ contains a simple closed curve enclosing a point of $M$ and having in common with the set $C_{1}+C_{2}$ exactly one point. This is impossible since neither $C_{1}$ nor $C_{2}$ is a cut point of $M$. Thus the supposition that $M_{1}$ is not connected has led to a contradiction.

Suppose $X$ and $Y$ are points belonging to different components of $M-T$. There exists an $\operatorname{arc} X Y$ which contains no point of $T$. Let $Y_{1}$ denote the first point in the order from $X$ to $Y$ which $X Y$ has in common with that component $M_{2}$ of $M-T$ which contains $Y$. Since $M$ is a continuous curve there exists a first point $X_{1}$ in the order from $Y_{1}$ to $X$ which the interval $Y_{1} X$ of $X Y$ has in common with $M-M_{2}$. Hence $X_{1}$ and $Y_{1}$ belong to the boundary of the same complementary domain of $M+T$ but to different components of $M-T$, which is impossible. Therefore the supposition that $X$ and $Y$ belong to different components of $M-T$ has led to a contradiction and Theorem VII is established.

† See R. L. Moore, A theorem concerning continuous curves, Bulletin of the American Mathematical Society, vol. 23 (1917).

$\ddagger$ Ibid. 
Theorem VIII. Suppose (a) $M$ is a continuous curve which contains no domain, (b) $A, B$ and $C$ are three distinct points, (c) $a_{1}$ and $a_{2}$ are two arcs with end points $A, B$ and $B, C$ respectively such that $a_{1}$ and $a_{2}$ each have property $\alpha$ with respect to $M$, (d) $B$ does not belong to $M$. Then there exists an arc from $A$ to $C$ which is a subset of a point set consisting of the sum of the arcs $a_{1}$ and $a_{2}$ together with a single complementary domain of $M$, such that arc $A C$ has property $\alpha$ with respect to $M$.

The case where $A$ and $C$ both belong to a point set consisting of a single complementary domain of $M$ together with its boundary, or where $A$ belongs to arc $a_{2}$, is trivial. If $A$ belongs to a complementary domain $D$ of $M$ let $H$ denote $\bar{D}$. If $A$ belongs to $M$ let $G_{1}$ denote the collection of all complementary domains of $M$ such that $A$ belongs to the boundary of each domain of the collection $G_{1}$, and let $H$ denote the point set consisting of the sum of all the domains of the collection $G_{1}$ together with their boundaries. Since the complementary domains of $M$ form a contracting sequence, $\dagger H$ is closed. If $a_{2}$ contains a point of $H$ let $E$ denote the first such point in the order from $C$ to $B$. Then $E$ belongs to the boundary $B_{1}$ of a domain $D_{1}$ of the collection $G_{1}$. There exists an arc from $A$ to $E$ which, except for end points, is a subset of $D_{1}$. Arc $A E$ plus the interval $E C$ of $a_{2}$ is an arc from $A$ to $C$ which satisfies the conditions of the theorem. If $a_{2}$ contains no point of $H$, let $G_{2}$ denote the collection of all the complementary domains of $M$ such that each domain of the collection $G_{2}$ either contains a point of $a_{2}$ or has a point of $a_{2}$ on its boundary. Let $K$ denote the point set consisting of the sum of all the domains of the collection $G_{2}$ together with their boundaries. By Theorem $\mathrm{V}$ the set $K$ is closed. Since $a_{2}$ contains no point in common with $H$, the point $A$ does not belong to $K$. Let $F$ denote the first point in the order from $A$ to $B$ which $a_{1}$ has in common with $K$. If $F$ belongs to $a_{2}$ then the sum of the intervals $A F$ and $F C$ of $a_{1}$ and $a_{2}$ respectively is an arc from $A$ to $C$ satisfying the conditions of the theorem. If $F$ does not belong to $a_{2}$ it belongs to the boundary $B_{2}$ of a domain $D_{2}$ of the collection $G_{2}$. Let $V$ denote the first point in the order from $C$ to $B$ which $a_{2}$ has in common with $B_{2}+D_{2}$. There exists an arc from $F$ to $V$ which except for end points is a subset of $D_{2}$. The sum of the intervals $A F$ and $V C$ of $a_{1}$ and $a_{2}$ respectively together with $\operatorname{arc} F V$ is an $\operatorname{arc}$ from $A$ to $C$ satisfying the conditions of the theorem and the proof is complete.

THEOREM IX. If $P$ is not a cut point of a continuous curve $M$ and $e$ is a positive number, there exists a circle $C$ enclosing $P$ and of diameter less than $e$ such that the set of all points of $M$, each at a distance from $P$ greater than $e$, lie in a connected subset of $M-C$.

† See K. L. Moore, Report on continuous curves from the viewpoint of analysis situs, loc. cit. 
Let $M_{1}$ denote the set of all points of $M$ each at a distance from $P$ greater than or equal to $e$. Let $G$ denote a collection of connected open subsets of $M$ no one of which contains $P$ but such that if $X$ is any point of $M_{1}$ then $X$ belongs to some subset $g_{X}$ of the collection $G$. There exists a finite subcollection $G_{1}$ of $G$ which contains all the points of $M_{1}$. Since $M-P$ is connected and any two points belonging to a connected open subset of a continuous curve can be joined by an arc $\dagger$ lying wholly in the open subset it follows that there exists a finite set $A$ of arcs, each lying within $M-P$, and such that the point set $M_{2}$ consisting of $M_{1}$ plus the arcs of the set $A$ is connected. Since $M_{2}$ does not contain $P$ there exists a circle enclosing $P$ which neither contains nor encloses any point of $M_{2}$ and the theorem is established.

TheOREM X. If $P$ is not a cut point of a bounded continuous curve $M$ which contains no domain and $e$ is a positive number, there exists a positive number $d_{e}$ such that if $A$ and $B$ are any two points, each at a distance from $P$ less than $d_{e}$, and $M-(A+B)$ is connected, then $A$ and $B$ are the extremities of an arc which has property $\alpha$ with respect to $M$ and which is of diameter less than $e$.

By Theorem IX, if $e$ is a positive number there exists a positive number $d_{e}$ such that the set of all points of $M$ each at a distance from $P$ greater than $e$ lie in a connected subset $M_{1}$ of $M$ such that no point of $M_{1}$ is at a distance from $P$ less than $d_{e}$. Let $C$ and $C_{1}$ denote circles with $P$ as center and of diameters $e$ and $d_{e}$ respectively. Let $I$ and $I_{1}$ denote the interiors of $C$ and $C_{1}$ respectively, and let $M_{1}^{*}$ denote that component of $M-I_{1}$ which contains $M_{1}$. Let $S$ denote the set of all points, let $S_{1}$ denote $S-I$, and let $S_{2}$ denote the set of all points $X$ such that $X$ belongs to a complementary domain of $M+C$ whose boundary is a subset of $M_{1}^{*}+C$. Let $K$ denote the set $M_{1}^{*}+S_{1}+S_{2}$ and let $D$ denote the complementary domain of $K$. Clearly $P$ is in $D$. Suppose $P_{1}$ and $P_{2}$ are two points of $D$ which belong to a complementary domain $D_{1}$ of $M$ but which can not be joined by an arc lying in $I \cdot D_{1}$. Let $a_{1}$ denote an arc from $P_{1}$ to $P_{2}$ which is a subset of $D$ and let $a_{2}$ denote an $\operatorname{arc}$ from $P_{1}$ to $P_{2}$ which is a subset of $D_{1}$. If $a_{2}$ is a subset of $C+I$ let $a_{n}$ denote $a_{2}$. If $a_{2}$ is not a subset of $C+I$ let $E_{1}$ denote the first point in the order from $P_{1}$ to $P_{2}$ which $a_{2}$ has in common with $a_{1}$ such that interval $P_{1} E_{1}$ of $a_{2}$ contains a point exterior to $C$ and let $F_{1}$ denote the last point in the order from $P_{1}$ to $E_{1}$ which $P_{1} E_{1}-E_{1}$ has in common with $a_{1}$. Let $J_{1}$ denote the simple closed curve which is the sum of the intervals $E_{1} F_{1}$ of $a_{1}$ and $E_{1} F_{1}$ of $a_{2}$. If $M_{1}^{*}$ is interior to $J_{1}$ there exists a complementary domain of $J_{1}+C$ common to $I$ and the exterior of $J_{1}$ such that its boundary $J_{1}^{*}$ contains the interval $E_{1} F_{1}$ of $a_{1}$. If $M_{1}^{*}$ is a subset of the exterior of $J_{1}$ there exists a complementary domain of $J_{1}+C$ common

$\dagger$ See R. L. Moore, Concerning continuous curves in the plane, loc. cit., Theorem 1. 
to $I$ and the interior of $J_{1}$ such that its boundary $J_{1}^{*}$ contains the interval $E_{1} F_{1}$ of $a_{1}$. In either case let $t_{1}$ denote the arc from $P_{1}$ to $E_{1}$ which is the sum of the interval $P_{1} F_{1}$ of $a_{2}$ and the $\operatorname{arc} E_{1} F_{1}$ of $J_{1}^{*}$ which contains a point of $C$. Let $W_{1}$ denote the last point in the order from $P_{1}$ to $P_{2}$ which $a_{2}$ has in common with $t_{1}$. Let $a_{3}$ denote the sum of the intervals $P_{1} W_{1}$ and $W_{1} P_{2}$ of $t_{1}$ and $a_{2}$ respectively. Arc $a_{3}$ contains no point of $M$. If $a_{3}$ is a subset of $C+I$ let $a_{n}$ denote $a_{3}$. If $a_{3}$ is not a subset of $C+I$ let $E_{2}$ denote the first point in the order from $P_{1}$ to $P_{2}$ which $a_{3}$ has in common with $a_{1}$ such that interval $P_{1} E_{2}$ of $a_{3}$ contains a point exterior to $C$, and let $F_{2}$ denote the last point in the order from $P_{1}$ to $E_{2}$ which $P_{1} E_{2}-E_{2}$ has in common with $a_{1}$. Let $J_{2}$ denote the simple closed curve which is the sum of the intervals $E_{2} F_{2}$ of $a_{1}$ and $E_{2} F_{2}$ of $a_{3}$. If $M_{1}^{*}$ is interior to $J_{2}$ there exists a complementary domain of $J_{2}-C$ common to $I$ and the exterior of $J_{2}$ such that its boundary $J_{2}^{*}$ contains the interval $E_{2} F_{2}$ of $a_{1}$. If $M_{1}^{*}$ is exterior to $J_{2}$ there exists a complementary domain of $J_{2}+C$ common to $I$ and the interior of $J_{2}$ such that its boundary $J_{2}^{*}$ contains the interval $E_{2} F_{2}$ of $a_{1}$. In either case let $t_{2}$ denote the arc from $P_{1}$ to $E_{2}$ which is the sum of the interval $P_{1} F_{2}$ of $a_{3}$ and the arc $E_{2} F_{2}$ of $J_{2}^{*}$ which contains a point of $C$. Let $W_{2}$ denote the last point in the order from $P_{1}$ to $P_{2}$ which $a_{3}$ has in common with $t_{2}$. Let $a_{4}$ denote the sum of the intervals $P_{1} W_{2}$ and $W_{2} P_{2}$ of $t_{2}$ and $a_{3}$ respectively. Arc $a_{4}$ contains no point of $M$. If $a_{4}$ is a subset of $C+I$ let $a_{n}$ denote $a_{4}$. If $a_{4}$ contains a point exterior to $C$ it is clear that after a finite number of operations just described one may obtain an arc $a_{n}$ from $P_{1}$ to $P_{2}$ which is a subset of $C+I$ and which contains no point of $M$. It follows that there exists an $\operatorname{arc} a_{m}$ from $P_{1}$ to $P_{2}$ which is a subset of $I \cdot D_{1}$ contrary to the assumption that $P_{1}$ and $P_{2}$ are points of $D_{1}$ which can not be joined by an arc of $I \cdot D_{1}$.

If $K$ is designated by a point and each point of $S-K$ is designated by a point, the set of elements thus obtained is an upper semi-continuous collection $\dagger$ of elements filling up the plane and is in one-to-one continuous correspondence $T$ with the surface $H$ of a sphere. For each point set $Q$ in $S$ let $T(Q)$ designate its image under $T$. Since any two points of $D$ belonging to a complementary domain $D_{i}$ of $M$ can be joined by an arc of $I \cdot D_{i}, T\left(D \cdot D_{i}\right)$

† See R. L. Moore, Concerning upper semi-continuous collections of continua, these Transactions, vol. 27 (1925), pp. 416-428. A collection $G$ of continua is said to be an $u p p e r$ semi-continuous collection if for each element $g$ of the collection $G$ and each positive number $e$ there exists a positive number $d$ such that if $x$ is any element of $G$ at a lower distance from $g$ less than $d$ then the upper distance of $x$ from $g$ is less than $e$. If $M$ is a point set and $P$ is a point, then by $l(P M)$ is meant the lower bound of the distance from $P$ to all the different points of $M$. If $M$ and $N$ are two point sets, then by $l(M N)$ is meant the lower bound of the values $l(P N)$ for all points $P$ of $M$, while by $u(M N)$ is meant the upper bound of these values for all points $P$ of $M$. The point set $M$ is said to be at the upper distance $u(M N)$ from the point set $N$ and is said to be at the lower distance $l(M N)$ from $N$. 
is a complementary domain of $T(K+M)$. If $A B$ is an arc in $D$ such that $T(A B)$ has property $\alpha$ with respect to $T(K+M)$, then $A B$ has property $\alpha$ with respect to $M$. By a slight modification of a theorem by $\mathrm{R}$. L. Moore $\dagger$ it may easily be seen that any two points belonging to $H-T(K)$ whose omission leaves $T(K+M)$ connected may be joined by an arc lying in $H-T(K)$ and which has property $\alpha$ with respect to $M$. Therefore Theorem $\mathrm{X}$ is established.

Theorem XI. If $M$ is a bounded continuous curve which contains no domain, and $A$ and $B$ are two points such that $M-(A+B)$ is connected, there exists an arc from $A$ to $B$ which has property $\alpha$ with respect to $M$.

If neither $A$ nor $B$ belongs to $M$ then R. L. Moore $\ddagger$ has shown how to construct an arc from $A$ to $B$ which does not disconnect $M$. From the nature of his construction, this arc has property $\alpha$ with respect to $M$. If $M$ contains no domain and $M-(A+B)$ is connected it follows from a slight modification of his construction that there exists an arc from $A$ to $B$ which has property $\alpha$ with respect to $M$.

Theorem XII. If $M$ is a bounded continuous curve which contains no domain and $K$ is a totally disconnected closed subset of $M$, there exists an acyclic continuous curve $T$ containing $K$, and such that (1) all, the end points of $T$ belong to $K$, and (2) the point set $M \cdot T$ is totally disconnected and $M-T$ is connected.

By Theorem IV, for each complementary domain $D_{i}$ of $M$ whose boundary $\delta_{i}$ contains two or more points of $K$, there exists an acyclic continuous curve $C_{i}$ whose end points are identical with the points of the set $K \cdot \delta_{i}$ and lying except for end points wholly within $D_{i}$. Let $K_{1}$ denote the point set $K+C_{1}+C_{2}+C_{3}+\cdots$. That $K_{1}$ is closed may be proved with the use of the fact that the complementary domains of $M$ form a contracting sequence. $\S$ By Theorem VI each component of $K_{1}$ is a continuous curve and not more than a finite number of components of $K_{1}$ are of diameter greater than a positive number. Suppose $K_{1}$ contains a simple closed curve $J$. Since $J \cdot M$ $=J \cdot K$ and $M-K$ is connected, then $M-J$ is a subset of one of the complementary domains of $J$ and hence $J$ is a subset of a point set consisting of a single complementary domain of $M$ plus its boundary which is impossible. Hence $K_{1}$ contains no simple closed curve.

By Theorems IX and X together with the fact that a closed and bounded

$\dagger$ Concerning paths that do not separate a given continuous curve, loc. cit.

$\ddagger$ Ibid.

$\S$ See R. L. Moore, Report on continuous curves from the viewpoint of analysis situs, loc. cit. 
point set has the Borel-Lebesgue property, there exists a sequence of sets of circular regions $G_{1}, G_{2}, G_{3}, \cdots$ having the properties that for each positive integer $i,(1) G_{i}$ is finite and covers $K$ and each region of $G_{i}$ contains at least one point of $K,(2)$ each region of the set $G_{i}$ is of diameter less than $1 / i,(3)$ if $g$ is a region of the set $G_{i}(i>1), \bar{g}$ lies interior to some region of the set $G_{i-1}$ and has the property that if $X_{1}$ and $X_{2}$ are any two points of $g$ whose omission does not disconnect $M$ then there exists an arc from $X_{1}$ to $X_{2}$ having property $\alpha$ with respect to $M$ and lying interior to each region of the set $G_{i-1}$ which contains $\bar{g}$.

For each positive integer $i$ let $N_{1 i}$ denote the point set obtained by adding to $K_{1}$ all the regions of the set $G_{i}$ together with their boundaries. If for all values of $i$ the set $N_{1 i}$ is connected, let $T$ denote the set of points common to the sets $N_{11}, N_{12}, N_{13}, \cdots$. If for some value of $i$ the set $N_{1 i}$ is not connected let $m$ denote the smallest positive integer such that $N_{1 m}$ is not connected. The point set $N_{1 m}$ has only a finite number $n$ of components. Let $L_{1}$ denote one of them. If $m$ is greater than 3 there exists a component $L_{2}$ of $N_{1 m}$ distinct from $L_{1}$ and two regions $g_{s}$ and $g_{t}$ of the set $G_{m-2}$ having in common a point $P$ not belonging to $M$ such that $g_{s}$ contains a point $X_{1}$ of the set $L_{1} \cdot K_{1}$ and $g_{t}$ contains a point $X_{2}$ of the set $L_{2} \cdot K_{1}$. By property (3) of the sets of circular regions, there exist arcs $P X_{1}$ and $P X_{2}$ each having property $\alpha$ with respect to $M$ and each lying in a single region of the set $G_{m-3}$. By Theorem VIII there exists an $\operatorname{arc} X_{1} X_{2}$ having property $\alpha$ with respect to $M$ and which is a subset of a point set consisting of the sum of the $\operatorname{arcs} P X_{1}$ and $P X_{2}$ together with a single complementary domain of $M$. For $m=1,2,3$ there exists an $\operatorname{arc} X_{1} X_{2}$ from a point $X_{1}$ of $L_{1} \cdot K_{1}$ to a point $X_{2}$ of $K_{1}-L_{1}$ which has property $\alpha$ with respect to $M$.

If $k$ is a component of $K_{1}$ then for each point $Z$ of $k$ add to $k$ all points $W$ such that $W$ lies within or on the boundary of some complementary domain of $M$ which contains $Z$ or has $Z$ on its boundary. Let $H_{k}$ denote the set thus obtained and let $H$ denote the point set obtained by adding together all points of all the sets $H_{k}$ for all components $k$ of $K_{1}$. Since each point of $H$ at a distance from every point of $K$ greater than a positive number $e$ lies either within or on the boundary of a complementary domain of $M$ of diameter greater than $e$ it follows that $H$ is closed. From the fact that $K$ is closed and totally disconnected and the fact that every infinite set of point sets whose sum is bounded has a limiting set, $\dagger$ it follows that the nondegenerate $\ddagger$ sets $H_{k}$ form a contracting sequence.

Let $H^{*}$ denote the point set obtained by adding together all points of all

† See R. G. Lubben, Concerning limiting sets in abstract spaces, loc. cit.

$\ddagger$ A point set containing but a single point is said to be degenerate. 
the sets $H_{k}$ for all components $k$ of $K_{1}$ such that $k$ is a subset of $L_{1}$. Since the components of $K_{1}$ and the complementary domains of $M$ form contracting sequences and each component of $K_{1}$ contains a point of $K$, therefore $H^{*}$ is closed. The point set $H^{*} \cdot K_{1}$ is a subset of $L_{1}$. Let $E_{1}$ denote the last point which the $\operatorname{arc} X_{1} X_{2}$ has in common with $H^{*}$, and let $E_{2}$ denote the first point which the interval $E_{1} X_{2}$ of $X_{1} X_{2}$ has in common with the set $\overline{H-H^{*}}$. If $E_{1}=E_{2}$ then $E_{1}$ does not belong to $K_{1}$. For suppose it does. Then since $E_{1}$ belongs to $M$ it belongs also to $K$. Hence $E_{1}$ does not belong to any set $H_{k}$ for any component $k$ of $K_{1}$ such that $K$ is a subset of $K_{1}-L_{1}$. Since $E_{1}$ belongs to $K$ it is interior to some region of the set $G_{m}$ belonging to $L_{1}$ and there exists a positive number $e$ such that the distance from $E_{1}$ to any point of $K_{1}-L_{1}$ is greater than $e$. Therefore since $E_{1}$ belongs to $\overline{H-H^{*}}$ there are infinitely many sets $H_{k}$, each of diameter greater than $e / 2$, which is impossible. Hence the supposition that $E_{1}=E_{2}$ and $E_{1}$ belongs to $K_{1}$ has led to a contradiction. Thus if $E_{1}=E_{2}$, then $E_{1}$ belongs to the boundaries of two distinct complementary domains $D_{1}$ and $D_{2}$ of $M$ such that the boundary of $D_{1}$ contains a point of $K$ belonging to $L_{1}$ and the boundary of $D_{2}$ contains a point of $K$ belonging to the set $N_{1 m}-L_{1}$. There exists in $\bar{D}_{1}$ an arc $Y_{1} E_{1}$ and in $\bar{D}_{2}$ an $\operatorname{arc} Z_{1} E_{2}$ such that (a) the point $Y_{1}$ belongs to $K_{1}$ and also to $K_{1} \cdot D_{1}$ if $D_{1}$ contains a point of $K_{1}$, (b) the point $Z_{1}$ belongs to $K_{1}$ and also to $K_{1} \cdot D_{2}$ if $D_{2}$ contains a point of $K_{1}$, (c) segments $Y_{1} E_{1}$ and $Z_{1} E_{2}$ have no point in common with $K_{1}$ or with $M$. If $E_{1} \neq E_{2}$ and $E_{1}$ belongs to $K_{1}$, let $Y_{1}$ denote $E_{1}$. If $E_{1} \neq E_{2}$ and $E_{1}$ does not belong to $K_{1}$, it belongs to the boundary $B_{1}$ of a complementary domain $D_{1}$ of $M$ such that $B_{1}$ contains a point of $K_{1} \cdot L_{1}$. There exists an $\operatorname{arc} Y_{1} E_{1}$ such that (a) the point $Y_{1}$ belongs to $K_{1}$ and also to $K_{1}-K$ if $D_{1}$ contains a point of $K_{1}$, (b) segment $Y_{1} E_{1}$ contains no point in common with $K_{1}$ or with $M$. If $E_{1} \neq E_{2}$ and $E_{2}$ belongs to $K_{1}$, let $Z_{1}$ denote $E_{2}$. If $E_{1} \neq E_{2}$ and $E_{2}$ does not belong to $K_{1}$, it belongs to the boundary $B_{2}$ of a complementary domain $D_{2}$ of $M$ such that $B_{2}$ contains a point of $K_{1}-L_{1}$. There exists an $\operatorname{arc} Z_{1} E_{2}$ such that (a) the point $Z_{1}$ belongs to $K_{1}$ and also to $K_{1}-K$ if $D_{2}$ contains a point of $K_{1}$, (b) segment $Z_{1} E_{2}$ contains no point in common with $K_{1}$ or with $M$. Let $a_{11}$ denote the arc which is the sum of the $\operatorname{arcs} Y_{1} E_{1}$ and $Z_{1} E_{2}$ and the interval $E_{1} E_{2}$ of the $\operatorname{arc} X_{1} X_{2}$. Then (1) the arc $a_{11}$ has property $\alpha$ with respect to $M,(2)$ for $m$ greater than 3 the set of points common to arc $a_{11}$ and $M$ which does not belong to $K_{1}$ is a subset of a point set consisting of the sum of two regions of the set $G_{m-3}$, (3) the end points $Y_{1}$ and $Z_{1}$ of arc $a_{11}$, which are the only points common to $a_{11}$ and to $K_{1}$, belong to different components of $N_{1 m}$. The number of components of $N_{1 m}+a_{11}$ is at least one less than the number of components of $N_{1 m}$. If $N_{1 m}+a_{11}$ is not connected, by treating the sets $K_{1}+a_{11}$ and $N_{1 m}+a_{11}$ in the same manner as 
that in which the sets $K_{1}$ and $N_{1 m}$ respectively were treated one may obtain an arc $a_{12}$ which satisfies with respect to $M, G_{m-3}, K_{1}+a_{11}$, and $N_{1 m}+a_{11}$, the same properties that arc $a_{11}$ satisfies with respect to $M, G_{m-3}, K_{1}$ and $N_{1 m}$ respectively. The number of components of the set $N_{1 m}+a_{11}+a_{12}$ is at least two less than the number of components of the set $N_{1 m}$. It is clear that by the addition to $K_{1}$ of a finite set $A_{1}$ of $\operatorname{arcs} a_{11}, a_{12}, a_{13}, \cdots, a_{1 k}(k<n)$, each arc $a_{1 j}$ of the set $A_{1}$ satisfying with respect to $M, G_{m-3}, K_{1}+\sum_{i=1}^{j-1} a_{1 i}$ and $N_{1 m}+\sum_{i=1}^{j-1} a_{1 i}$ the same properties that arc $a_{i(j-1)}$ satisfies with respect to $M$, $G_{m-3}, K_{1}+\sum_{i=1}^{j-2} a_{1 i}$ and $N_{1 m}+\sum_{i=1}^{j-2} a_{1 i}$ respectively, one may obtain a point set $K_{2}$ which has the following properties: (1) $K_{2}$ is closed and contains no simple closed curve, (2) if $P$ and $Q$ are any two points of $K_{2}$ belonging to the boundary of a complementary domain $D$ of $M$ then $P$ and $Q$ are the extremities of an arc belonging to $K_{2}$ such that segment $P Q$ is a subset of $D,(3)$ the set $K_{2}+N_{1 m}$ is connected, (4) the set $M \cdot K_{2}$ is totally disconnected, (5) each component of $K_{2}$ is an acyclic continuous curve, and (6) $K_{2}$ contains no cut point of $M$. That $K_{2}$ has properties (1) and (2) is clear from the facts (a) $A_{1}$ is finite, (b) $K_{1}+a_{11}$ has properties (1) and (2), (c) if $K_{1}+\sum_{i=1}^{j-1} a_{1 i}(j<k-1)$ has properties (1) and (2) then $K_{1}+\sum_{i=1}^{j} a_{1 i}$ has them also. The point set $K_{2}$ has property (3) since the number of components of $N_{1 m}$ is $n$ and the number of components of $N_{1 m}+\sum_{i=1}^{r} a_{1 i}(r<k-1)$ is at least one less than the number of components of $N_{1 m}+\sum_{i=1}^{r-1} a_{1 i}$. The set of points common to $M$ and the set $A_{1}$ of arcs is closed and totally disconnected, hence $K_{2}$ has property (4). By Theorem VI the set $K_{2}$ has property (5), and since no arc of the set $A_{1}$ contains a cut point of $M$ the set $K_{2}$ has the property (6). Add $K_{2}$ to each of the sets $N_{11}, N_{12} ; N_{13}, \cdots$ and denote the resulting sets by $N_{21}, N_{22}, N_{23}, \cdots$ respectively. If for each value of $i, N_{2 i}$ is connected, let $T$ denote the set of all points common to the sets $N_{21}, N_{22}, N_{23}, \ldots$. If, for some value of $i, N_{2 i}$ is not connected, let $n$ denote the smallest positive integer such that $N_{2 n}$ is not connected. Let $r$ denote the number of components of $N_{2 n}$. By the addition to $K_{2}$ of a finite set $A_{2}$ of $\operatorname{arcs} a_{21}, a_{22}, a_{23}, \cdots, a_{2 j}(j<r)$, each arc $a_{2 k}$ of the set $A_{2}$ satisfying with respect to $M, G_{n-3}, K_{2}+\sum_{i=1}^{k-1} a_{2 i}$ and $N_{2 n}+\sum_{i=1}^{k-1} a_{2 i}$ $(k<r)$ the same properties that arc $a_{11}$ satisfies with respect to $M, G_{m-3}, K_{1}$ and $N_{1 m}$ respectively, one may obtain a point set $K_{3}$ which satisfies the same properties with respect to $M, G_{n-3}, N_{2 n}$ that $K_{2}$ satisfies with respect to $M$, $G_{m-3}$ and $N_{1 m}$ respectively. Suppose the process is continued indefinitely. Let $K^{*}$ denote the set $K_{1}+K_{2}+K_{3}+\cdots$. The set $K^{*}$ is connected and the set $M \cdot K^{*}$ is closed. For suppose $W$ denotes a limit point of $M \cdot K^{*}$ which does not belong to $M \cdot K^{*}$. The point $W$ does not belong to $K$, and since for any positive number $e$ there exists a positive integer $t$ such that no arc of the set $A_{j}(j>t)$ contains a point of $M-K_{t-1}$ at a distance from every point of $K$ 
greater than $e$, it follows that $W$ belongs to $K_{t-1}$ and hence to $M \cdot K^{*}$ contrary to hypothesis. Therefore $M \cdot K^{*}$ is closed. Since for each positive integer $i$ the set $K_{i}$ contains in common with $M$ only a totally disconnected set of points, the point set $M \cdot K^{*}$ is totally disconnected. From the fact that each arc of the set $A_{i}$ for all values of $i$ has property $\alpha$ with respect to $M$ and the fact that $M-K$ is connected it follows that $M \cdot K^{*}$ contains no cut point of $M$.

If $\beta_{i}$ is the boundary of a complementary domain $D_{i}$ of $M$ and the set of points $\bar{D}_{i} \cdot K^{*}$ is not an acyclic continuous curve whose end points are identical with the set $\beta_{i} \cdot K^{*}$, there exists by Theorem IV an acyclic continuous curve $C_{i}$ whose end points are identical with the set $\beta_{i} \cdot K^{*}$ and which, except for end points, is a subset of $D_{i}$. Let $T_{1}$ denote the set formed from $K^{*}$ by replacing the set $D_{i} \cdot K^{*}$ by $C_{i}$ for each value of $i$. The point set $T_{1}$ is closed and connected and has the property that if $X$ and $Y$ are any two points of $T_{1}$ belonging to the boundary of a complementary domain $D$ of $M$ then $X$ and $Y$ are the extremities of an arc belonging to $T_{1}$ such that segment $X Y$ is a subset of $D$. That $T_{1}$ is a continuous curve may be seen from the fact that any point of $T_{1}$ not belonging to the totally disconnected set $M \cdot T_{1}$ lies in some complementary domain of $M$, together with the fact that a continuous curve can not fail to be connected im kleinen at only a totally disconnected set of points. $\dagger$

Suppose there exists an end point $P$ of $T_{1}$ which does not belong to $K$. Then $P$ belongs to $M$ and hence to $K^{*}$ and there exists a positive integer $r$ such that $P$ belongs to $K_{r}$. By a theorem of G. T. Whyburn $\ddagger$ and the fact that each nondegenerate component of $K_{r}$ is an acyclic continuous curve, all of whose end points belong to $K$, it follows that there exists an arc $c_{r}$ lying in $K_{r}-K$ whose end points belong to $M$ and such that $c_{r}$ contains $P$ as an interior point. For each segment $c_{i}$ of $c_{r}-M$ whose end points belong to $M$ let $b_{i}$ denote a segment of $T_{1}-M$ such that the end points of $b_{i}$ are the end points of $c_{i}$. The point set consisting of the sum of the segments $b_{i}$ together with the set $M \cdot c_{r}$ is an arc lying in $T_{1}$ and containing $P$ as interior point which contradicts the assumption that $P$ is an end point of $T_{1}$. Hence the end points of $T_{1}$ all belong to $K$.

Suppose $J$ is a simple closed curve lying in $T_{1}$. The curve $J$ contains a point of $M-K$, otherwise $J$ is a subset of a point set consisting of a single complementary domain $D$ of $M$ together with its boundary, contrary to the fact that $\bar{D} \cdot T_{1}$ contains no simple closed curve. The curve $J$ contains a point of $K$. For suppose it does not. There exists a positive integer $s$ such that $M \cdot J$

† See R. L. Moore, Report on continuous curves from the viewpoint of analysis situs, loc. cit. $\ddagger$ Concerning continua in the plane, loc. cit. 
is a subset of $M \cdot K_{s}$. Since $J$ separates $M$ there exists a point of $M-K_{s}$ interior to $J$ and a point of $M-K_{s}$ exterior to $J$. For each segment $c_{i}$ of $J-M$ whose end points belong to $M$ let $b_{i}$ denote a segment lying in $K_{s}-M$ and such that the end points of $b_{i}$ are the end points of $c_{i}$. The point set consisting of the sum of the segments $b_{i}$ together with the set $M \cdot J$ is a connected set of points which is a subset of a single component $k$ of $K_{s}$. Hence $k$ separates $M$. Since $K_{s}$ contains no cut point of $M$ and has the properties (1) $K_{s}$ contains no simple closed curve, (2) $M \cdot K_{8}$ is totally disconnected, (3) any two points of $K_{s}$ belonging to the boundary $B$ of a complementary domain $D$ of $M$ are the end points of a segment lying in $D \cdot K_{s}$, therefore any component of $K_{s}$ is an acyclic continuous curve which satisfies with respect to $M$ the same properties that $T$ satisfies with respect to $M$ in Theorem VII. Hence $k$ does not separate $M$ and the supposition that $J$ contains no point of $K$ has led to a contradiction.

For each positive integer $i$ let $S_{i}$ denote the sum of the regions of the set $G_{i}$ together with their boundaries. That the set of all junction points $\dagger$ of $T_{1}$ not belonging to $S_{i}$ is finite may be shown as follows. Since $T_{1}$ is a continuous curve, for $j>i$ there are not more than a finite number of components of $T_{1}-S_{j}$ each containing a point of $T_{1}-S_{i}$. Since all the end points of $T_{1}$ belong to $K$ and any simple closed curve of $T_{1}$ contains a point of $K$, then each component of $T_{1}-S_{j}$ together with its limit point, which contains a point of $T_{1}-S_{i}$, is an acyclic continuous curve whose end points, finite in number, all belong to $S_{i}$. Let $L$ denote the set of all junction points of $T_{1}$. For each positive integer $i$ there are only a finite number of components of the set $T_{1}-\left(L+S_{i+1}\right)$ each of which contains a point of $T_{1}-S_{i}$. Suppose there exist a positive integer $m$ and a component $r$ of $T_{1}-\left(L+S_{m+1}\right)$ such that for each positive integer $n$ there exists a simple closed curve of $T_{1}$ which contains $r$ but which contains no point of $M-S_{n}$. Since each simple closed curve of $T_{1}$ contains a point of $M-K$ it follows that there exists a sequence $J_{1}, J_{2}, J_{3}, \cdots$ of simple closed curves of $T_{1}$ and a subsequence $S_{1}^{*}, S_{2}^{*}, S_{3}^{*}, \cdots$ of the sequence $S_{1}, S_{2}, S_{3}, \cdots$ such that for each $i, J_{i}$ contains $r$ and at least one point of $M-S_{i+1}^{*}$ but contains no point of $M-S_{i}^{*}$. Let $J_{1}^{*}, J_{2}^{*}, J_{3}^{*}, \cdots$ denote a subsequence of the sequence $J_{1}, J_{2}, J_{3}, \cdots$ having a sequential limiting set $N^{*}$. Since for each $i$ the curve $J_{i}^{*}$ contains $r$ the set $N^{*}$ separates the plane. Suppose $N^{*}$ contains

† See R. L. Moore, Concerning triods in the plane and the junction points of plane continua, Proceedings of the National Academy of Sciences, vol. 14 (1928). If $P$ is a point of a continuous curve $N$ and $K$ is a domain containing $P$ such that $P$ is a cut point of the component of $N \cdot K$ which contains $P$, and furthermore there exist three arcs $P A_{1}, P A_{2}$, and $P A_{3}$ which lie in $N$ and have only the point $P$ in common, then $P$ is said to be a junction point of $N$. The continuum $P A_{1}+P A_{2}+P A_{3}$ is called a triod and the point $P$ is its emanation point. 
a point $Q$ belonging to $M-K$. There exists a positive integer $m$ such that $Q$ does not belong to $S_{m}$. It follows that $Q$ belongs to all but a finite number of the simple closed curves of the sequence $J_{1}, J_{2}, J_{3}, \ldots$. This is impossible, hence $N^{*}$ contains no point of $M-K$. Since $N^{*}$ is connected im kleinen at every point not belonging to $K$, then $N^{*}$ is a continuous curve. $\dagger$ Therefore by a theorem of R. L. Moore, $\ddagger N^{*}$ contains a simple closed curve. This is impossible since $N^{*}$ contains no point of $M-K$ and the supposition that for each positive integer $n$ there exists a simple closed curve of $T_{1}$ which contains $r$ but which contains no point of $M-S_{n}$ has led to a contradiction. Hence if $r$ is a component of $T_{1}-\left(L+S_{m}\right)$ there exists a positive integer $n$ such that every simple closed curve of $T_{1}$ which contains $r$ contains a point of $M-S_{n}$. It follows then that there exists a subsequence $S_{1}^{* *}, S_{2}^{* *}, S_{3}^{* *}, \cdots$ of the sequence $S_{1}, S_{2}, S_{3}, \cdots$ such that for each positive integer $i$, if $J$ is a simple closed curve of $T_{1}$ which is not a subset of $S_{i}$, then $J$ contains a point of $M-S_{i}^{* *}$. Suppose there exists a component of $T_{1}-\left(L+S_{2}\right)$ which is not a subset of $S_{1}$ but which is a subset of a simple closed curve $J$ lying in $T_{1}$. Let $P$ denote a point of $J$ belonging to $M-S_{1}^{* *}$. If $P$ is not a junction point of $T_{1}$ there exists a component $t$ of $J-(L+K)$ containing $P$. If $P$ is a junction point of $T_{1}$ it is a limit point of the set $M \cdot J$. For suppose there exists a segment $s_{1}$ of $(J-M)+P$ which contains $P$. Since $P$ does not belong to $K$ there exists a positive integer $e$ such that $P$ belongs to $K_{e}$ but not to $K_{e-1}$. Thus $P$ is an interior point of some arc of the set $A_{e-1}$. Since for any complementary domain $D$ of $M$ the set $\bar{D} \cdot T_{1}$ is an acyclic continuous curve whose end points are identical with the set $T_{1}(\bar{D}-D)$ it follows that the two components of $s_{1}-P$ belong to different complementary domains $D_{1}$ and $D_{2}$ of $M$ and that $P$ is both a junction point of $T_{1}$ and a boundary point of a complementary domain of the point set $\bar{D}_{1}+\bar{D}_{2}$. This is impossible since $P$ is interior to some arc of the set $A_{e-1}$, each arc of which has property $\alpha$ with respect to $M$ and the supposition that there exists a segment of $(J-M)+P$ which contains $P$ has led to a contradiction. It follows then that since $L-S_{1}^{* *}$ is a finite set of points there exists a point $Q$ belonging to $M \cdot J-S_{1}^{* *}$ which is not a junction point of $T_{1}$. Let $t$ denote the component of $J-(L+K)$ which contains $Q$. The component $t$ is either a segment of the curve $J$ or the curve minus a single point. Subtract $t$ from $T_{1}$. Let $T_{1}^{*}$ denote the point set $T_{1}-\sum_{i=1}^{i_{1}} t_{i}$ where for each positive integer $k\left(k \leqq i_{1}\right),(1) t_{k}$ is either a segment of a simple closed curve or a simple closed curve minus a point such that the curve is a subset of $T_{1}-\sum_{i=1}^{k-1} t_{i}$ and contains a component of the set $T_{1}-\left(L+S_{2}\right)$, (2) $t_{k}$

$\dagger$ See R. L. Moore, Report on continuous curves from the viewpoint of analysis situs, loc. cit. $\ddagger$ Concerning continuous curves in the plane, loc. cit. 
contains a point of $M-S_{1}^{* *}$, (3) if $t_{k}$ is a segment its end points belong to the point set consisting of $K$ together with the junction points of $T_{1}-\sum_{i=1}^{k-1} t_{i}$ and if $t_{k}$ is not a segment then $t_{k}$ plus a single point of $K$ is a simple closed curve, (4) $t_{k}$ contains no point of $K$ nor any junction point of $T_{1}-\sum_{i=1}^{k-1} t_{i}$, and further such that the point set $T_{1}^{*}$ contains no simple closed curve which is not a subset of $S_{1}$. In general let $T_{j}^{*}$ denote the point set $T_{j-1}^{*}-\sum_{i=1}^{i_{j}} t_{i}$, where for each positive integer $k\left(k \leqq i_{j}\right)$, (1) $t_{k}$ is either a segment of a simple closed curve or a simple closed curve minus a point such that the curve is a subset of $T_{j-1}^{*}-\sum_{i=1}^{k-1} t_{i}$ and contains a component of the set $T_{1}-\left(L+S_{j+1}\right)$, (2) $t_{k}$ contains a point of $M-S_{j}^{* *}$, (3) if $t_{k}$ is a segment its end points belong to the point set consisting of $K$ together with the junction points of $T_{j-1}^{*}-\sum_{i=1}^{k-1} t_{i}$ and if $t_{k}$ is not a segment then $t_{k}$ plus a single point of $K$ is a simple closed curve, (4) $t_{k}$ contains no point of $K$ nor any junction point of $T_{j-1}^{*}-\sum_{i=1}^{k-1} t_{i}$, and further such that the point set $T_{j}^{*}$ contains no simple closed curve which is not a subset of $S_{j}$.

Let $T$ denote the set of points common to the sets $T_{1}^{*}, T_{2}^{*}, T_{3}^{*}, \cdots$. The continuum $T$ contains no simple closed curve. Since (a) for each positive integer $i$ the set $T_{i}^{*}-T_{i+1}^{*}$ consists of a finite number of components, each of which is a subset of $S_{i}$, (b) the continuous curve $T_{1}$ has the property that every simple closed curve lying in $T_{1}$ contains a point of $K$, it follows that $T$ is connected im kleinen at every point of $T-K$ and hence $T$ is a continuous curve. Since $M \cdot T$ is a subset of $M \cdot K^{*}$ the set $M \cdot T$ is totally disconnected and contains no cut point of $M$.

Suppose there exist two points $X$ and $Y$ which belong to the boundary $B$ of a complementary domain $D$ of $M$ such that $X$ and $Y$ are not the extremities of any arc belonging to $T$ and lying, except for end points, within $D$. There exists an arc $X Y$ lying within $\bar{D} \cdot T_{1}$ such that segment $X Y$ is a subset of $D$. There exist two positive integers $d$ and $e$ such that $T_{d}^{*}-\sum_{i=1}^{e} t_{i}$ contains segment $X Y$ and $T_{d}^{*}-\sum_{i=1}^{e+1} t_{i}$ does not. Hence $\left(T_{d}^{*}-\sum_{i=1}^{e} t_{i}\right)$ $-\left(T_{d}^{*}-\sum_{i=1}^{e+1} t_{i}\right)$ contains a junction point of $T_{d}^{*}-\sum_{i=1}^{e} t_{i}$ contrary to the fact that for any two positive integers $r$ and $s\left(T_{r}^{*}-\sum_{i=1}^{s} t_{i}\right)-\left(T_{r}^{*}-\sum_{i=1}^{s+1} t_{i}\right)$ contains no junction point of $T_{r}^{*}-\sum_{i=1}^{s} t_{i}$. Hence the supposition that $X$ and $Y$ are not the extremities of any arc belonging to $T$ and lying, except for end points, within $D$ has led to a contradiction. It follows then by Theorem VII that $M-T$ is connected.

Suppose there exists an end point $P$ of $T$ which does not belong to $K$. Since $P$ is not an end point of $T_{1}$ and for any positive integer $r$ there are only a finite number of components of the set $T_{1}-\left(L+S_{r+1}\right)$ each of which con-

† See R. L. Moore, Report on continuous curves from the viewpoint of analysis situs, loc. cit. 
tains a point of $T_{1}-S_{r}$ it follows that there exist two positive integers $d$ and $e$ such that $P$ is an end point of $T_{d}^{*}-\sum_{i=1}^{e+1} t_{i}$ but not an end point of $T_{d}^{*}-\sum_{i=1}^{c} t_{i}$. Since $P$ does not belong to $K,\left(T_{d}^{*}-\sum_{i=1}^{e} t_{i}\right)-\left(T_{d}^{*}-\sum_{i=1}^{e+1} t_{i}\right)$ is a segment lying in a simple closed curve which is a subset of $T_{d}^{*}-\sum_{i=1}^{e} t_{i}$. Hence the point $P$ is a junction point of $T_{d}^{*}-\sum_{i=1}^{e} t_{i}$ and an end point of $T_{d}^{*}-\sum_{i=1}^{e+1} t_{i}$. This contradicts the fact that no junction point of a continuous curve $N$ is an end point of $N-t$, where $t$ is a segment lying in $N$. Hence all the end points of $T$ belong to $K$ and Theorem XII has been established.

UNIVERSITY OF TEXAS,

Austrn, TExas 